\title{
MANUFACTURING SIMULATION: COMPUTER AIDED TOLERANCING FOR PROCESS PLANNING
}

\author{
Bouaziz, Z. \& Masmoudi, F. ${ }^{* *}$ \\ ${ }^{*}$ Unit of Mechanics, Solids, Structures and Technological Development, \\ Ecole Supérieure des Sciences et Techniques, BP 56 Beb Mnara, 1008 Tunis, Tunisia \\ ** Unit of Mechanics, Modeling, and Production, Ecole Nationale d'Ingénieurs de Sfax, \\ Route de Sokra Km 3.5 BP W, 3038 Sfax, Tunisia \\ E-Mail: zoubeir.bouaziz@enis.rnu.tn
}

\begin{abstract}
The simulation of the process planning is a decisive stage in the manufacturing process of a set of parts. This simulation is generally carried out by the calculation of the working dimensions (WD) according to the blueprint dimensions (BPD), to the means of production and to the machining process. The calculation and the generating of the working dimensions can be done by using different methods such as the transfer method, the dispersion method and the tolerance chart method.

This article presents a system of automatic generating of the working dimensions (WD) through the tolerance chart method. From the blueprint dimensions and from the process planning proposed, the system allows the automatic generating of three graphs: the blueprint dimensions graph, the blueprint dimensions and the stock removal graph, as well as the working dimensions graph. After the drawing of the two last graphs, the dimensional chains appear in the matrix from: $Y=P X$.

At last, the calculation of the tolerance intervals for each dimension is done from the resolution of an equation system according to the minimum economic tolerances imposed by the manufacturing process and to the weight assigned to the tolerances.

(Received in July 2005, accepted in October 2005. This paper was with the authors 1 month for 1 revision.)
\end{abstract}

Key Words: Manufacturing Simulation, Working Dimension, Dispersions

\begin{tabular}{ll}
\multicolumn{2}{l}{ Nomenclature } \\
BPD & Blueprint Dimensions \\
$\mathrm{SR}$ & Stock removal \\
$\mathrm{WD}$ & Working Dimensions \\
$\mathrm{SM}$ & Surface to machining \\
$\mathrm{Y}_{\mathrm{i}}$ & BPD Vector \\
$\mathrm{X}_{\mathrm{j}}$ & WD Vector \\
$\mathrm{P}$ & Matrix of passing from BPD to WD \\
$\mathrm{W}_{\mathrm{i}}$ & Tolerance weight \\
$\mathrm{b}_{\mathrm{i}}$ & Imposed tolerance \\
$\mathrm{e}_{\mathrm{i}}$ & Minimum economic tolerance \\
$\mathrm{S}_{\mathrm{i}}$ & Machined surface number $i$ \\
$\mathrm{~m}$ & Number of WD \\
$\mathrm{n}$ & Number of surfaces for machining
\end{tabular}

\section{INTRODUCTION}

The manufacturing process of an industrial product is defined by a set of stages which starting from a need allow, the achievement of this product. The design office deals with the 
conception, the process office with the preparation until the manufacturing, and the workshop achieves the product. Among the principal stages achieves from the preparation to the manufacturing, we find generation of the process planning, the machining simulation and the generation of the machining parameters.

The manufacturing simulation principally consists in validating the process planning through the calculation of the working dimensions according to the functional dimensions and to the means of productions [1]. For this, our study shows that there exit different methods for the working dimensions calculation, namely the dimensional chains method, the transfer method, the dispersion method [2-3] and the tolerance chart method [4].

The paper developed by [5] describes a tool for functional tolerance specification which supports the user in automatically proposing geometric tolerance types where the user only has to give in the tolerance values. Apart from this semi-automatic tolerance type specification, manual specification is still possible.

A computer aided tolerance analysis tool is presented to reference [6] that assists the designer in evaluating worst case quality of assembly after tolerances have been specified. In tolerance analysis calculations, sets of equations are generated. The number of equations can be restricted by using a minimum number of points in which quality of assembly in calculated.

Jami and al. [7] development a application of a geometric dimension and tolerance (GD\&T) model for use in both design and process planning. The model meets criteria for computability and compatibility with the Y14.5M standard. The model is first used to capture the designers GD\&T scheme on a feature based design model, validate its completeness, and then transfer the GD\&T to machining features extracted automatically by feature recognition. The model is based on relative degrees of freedom of geometric entities: feature axes, edges, faces and features-of-size. Dimension graphs are created based on the degrees of freedom for each control direction.

Yeo and al. [8] presents an approach that is capable of determining the optimal production sequence and its optimal process tolerance to achieve the minimum production costs. A new mathematical model, representing the cost-tolerance relationships and showing significant accuracy improvement over existing models, is introduced to allocate process tolerance.

The study presented in this paper consists in explaining a model of simulation and of the automatic generation of the working dimensions, basing on the tolerance chart method. In fact this method is based on the presentation of the different links between the condition imposed by the design office and the condition imposed by the process office in order to generate the WD. Each WD must be defined between a positioning surface and a machined surface [9-10].

The relations between BPD- WD and the machined surfaces are presented in the form of graphs: BPD graph, BPD graph and stock graph, and WD graph. The principle of the study done in this way is the presentation of an automatic model, allowing generating the three graphs so as to present the dimensional chains and to make the calculation of WD.

In the first part of this article we will present the simulation principle of the process planning. We will explain, afterwards, the working dimensions modelling with the tolerance chart method. We will start by presenting the method principle, and then we will present the model of the automatic generation of the dimension graphs in order to present the dimensional chains and the working dimensions model. We will finally set out in detail the stages of the working dimensions calculation for an example of a piece proposed by the collaborating society. 


\section{PROCESS PLANNING SIMULATION}

The process planning simulation is a very important operation in the elaboration of the product manufacturing file of a mass produced part. The design office establishes the definition drawing on which are mentioned all the dimensions and the functional specifications. On the other hand, the process office establishes the post project of the manufacturing study and so, the simulation comes to approve or disapprove the post-project of the manufacturing study as shown in figure1.

The object of the process planning simulation is to check the post-projects, to optimize the means of manufacturing and to calculate the working dimensions [1, 11]. Among the most efficient simulation methods we find the simulation through the tolerance chart method [910]. This method consists in giving the design office data the process office results as well as the process planning, the stock removal, and the tolerance in a chart. The values of the working dimensions and their tolerances will be added on the chart as the graph aided simulation goes on.

The objective of this research paper is to simulate the process planning and to integrate it in a system of Computer Aided Manufacturing CAM.

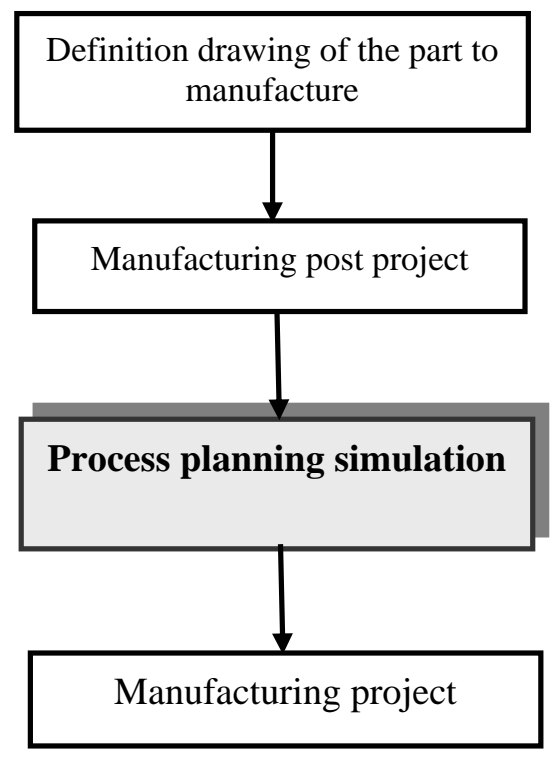

Figure 1: Manufacturing process.

\section{WORKING DIMENSIONS MODELLING WITH THE TOLERANCE CHART METHOD}

\subsection{The method principle}

The tolerance chart method [9-10] consists in presenting the design office data, the process office results, as well as the process planning, the stock removal and the tolerance in a chart. The working dimensions values and their tolerances will be added on the chart as the graph aided simulation goes on. This method makes use of three types of graphs: the first is the "blueprint dimensions", the second is the "blueprint and stock removal dimensions" and the third is the "working dimensions". Once the graphs are drawn, we determine the WD as well as their Tolerance Intervals TI. 


\subsection{Blueprint dimension graph}

From the graph theory, $n$ vertices and $n$ - 1 links form a graph structure if these vertices are all connected, or there is no circuit or isolated vertex [4, 12]. There are $n$ surfaces and $n-1$ Blueprint dimensions in a blueprint, all surfaces are dimensioned, so a graph can be generated form the blueprint dimensions. In a blueprint graph, the root is arbitrary. The algorithm (Algorithm 1) for generating a blueprint graph is as follows:

Step1: Set $i \leftarrow 1$

Step2: If the left surface of $Y_{i}$ has not been created as a vertex, create it.

Step3: If the right surface of $Y_{i}$ has not been created as a vertex, create it.

Step4: Connect the left and the right surfaces (vertices) with a solid line, and mark the link as $Y_{i}$.

Step5: If $i \neq(n-1)$, set $i \leftarrow i+1$, and go to step 2; otherwise, stop.

\subsection{Blueprint dimension and stock removal graph}

A blueprint graph can be extended to form another graph by adding stock removals on the appropriate vertices, and this new graph is called a blueprint stock removal graph.

A blueprint stock removal graph can be obtained by the following algorithm (Algorithm 2).

Step1: Create the blueprint graph firstly by use of Algorithm 1.

Step2: Set $m \leftarrow 1$.

Step3: For sequence No. $j$ :

Step 3.1: if the line to the locating surface of $Y_{i}$ is a dashed line, change it to be solid.

Step 3.2: if the line to the machined surface $S_{k}$ of $Y_{i}$ is a dashed line, change it to be solid.

Step 3.3: generate a new vertex $S_{j k+1}$, connect $S_{j k}$ and $S_{j k+1}$ with a dashed line, and mark the line as $Y_{j}$.

Step4: If $j \neq 1$, set $j \leftarrow j+1$ and $i \leftarrow i+1$, and go to step 3; otherwise, stop.

\subsection{Working dimension graph}

The third graph can be created from the working dimensions with the same vertices of a blue print stock removal graph (without dashed lines). This graph is called a $X_{i}$ graph and the algorithm (Algorithm 3) for generating a $X_{i}$ graph is as follows:

Step1: Create to locating surface $\left(S_{s}\right)$ of $X_{1}$ as the first vertex and this vertex is the root of the graph. Set $j \leftarrow 1$.

Step2: Create a vertex corresponding to the machined surface of $X_{j}$, mark the vertex as $S_{k}$ (if the surface $S$ has not been crated before, $k \leftarrow s$; otherwise, $k \leftarrow k-1$ ). Draw a line with an arrow from the locating surface (tail) to the machined surface $S_{k}$ (herd), and mark the directed line ( $\operatorname{arc})$ as $X_{j}$.

Step3: If $j \neq m$, set $j \leftarrow j+1$, and go to step 2; Otherwise, stop.

The graph generated from Algorithm 3 is a graph, so there are $m$ lines and $m+1$ vertices. The $m+1$ vertices of a $X$ graph are the same vertices of the corresponding blueprint stock removal graph because they are the same machined surfaces from the tolerance chart. One big difference between a $X$ graph and blueprint stock removal graph is that the former is a directed graph, while the latter is a non directed graph. 


\subsection{Linear equations for mean sizes of working dimensions}

In general, the procedure for manual tolerance charting is to assign tolerances and mean stock removals firstly, then to calculate the mean sizes of working dimensions. The tolerances have been obtained by the linear programming model, while the mean stock removals are determined from a handbook in the system. Now that $Y_{j}(j=1,2, \ldots, m)$ are known (except those solid stock removals) a blueprint stock removal graph is formed with the known, but a $X$ graph consists of all the unknowns. A blueprint stock removal graph (without dashed lines) has $\mathrm{m}$ lines, so $\mathrm{m}$ circuits (dimensional chains) can be identified, and $\mathrm{m}$ linear equations can be formed from the $m$ distinct dimensional chains. There are $m$ mean working dimensions in question, so the solution of the linear equations is the mean working dimensions required.

If the $\mathrm{m}$ dimensional chains of both blueprint dimensions and stock removals are renumbered, then the linear equations can be formulated as follows:

$$
Y=[P] \cdot X
$$

where $Y=\left(Y_{1}, Y_{2}, \ldots, Y_{m}\right)^{t}$ is a $m \times 1$ vector, its elements are either the mean stock removals or the mean $\mathrm{B} / \mathrm{P}$ dimensions, corresponding to the appropriate dimensional chains.

$X=\left(X_{1}, X_{2}, \ldots, X_{m}\right)^{t}$ is a $m \times 1$ vector of the unknowns, that is, the mean sizes of the $P=\left[p_{i j}\right]$ and working dimensions.

$$
p_{i j}=\left\{\begin{array}{cl}
1 & \text { if } X_{j} \text { is a positive constituent link of the } i^{t h} \text { dimensional chain } \\
-1 & \text { if } X_{j} \text { is a negative constituent link of the } i^{t h} \text { dimensional chain } \\
0 & \text { otherwise, } \quad i, j=1,2, \ldots, m
\end{array}\right.
$$

In fact, equation (1) correspond to $m$ distinct dimensional chains in the $X$ graph by adding $m$ distinct lines of the blueprint stock removal graph. So the equations must have a solution and the solution must be unique. The unique solution to equations (1) is: $X=[P]^{-1} \cdot Y$.

\subsection{Linear programming model for tolerance intervals}

The tolerance intervals problem for tolerance charting can be summarized to make each tolerance as large as possible subject to the BPD and process capability. This can be expressed as a linear programming model as follows:

Maximize

$$
W \cdot T
$$

Subject to

$$
\begin{aligned}
& A T \leq B \\
& T \geq E
\end{aligned}
$$

Where $W=\left[w_{1}, w_{2}, \ldots, w_{m}\right], w_{i}$ is the weight assigned for the tolerance $t_{i}$,

$T=\left[t_{1}, t_{2}, \ldots ., t_{m}\right]^{t}$ is vector tolerance,

$A=\left[a_{i j}\right]$ and

$$
\mathrm{a}_{\mathrm{ij}}=\left\{\begin{array}{l}
1 \text { if } X_{j} \text { is a constituent link of the } i^{\text {th }} \text { dimensional chain } \\
0 \text { otherwise, } \quad i, j=1,2, \ldots, m
\end{array}\right.
$$

There are $m$ dimensional chains, and these dimensional chains are numbered as $1,2, \ldots$, $m$, so $A$ is $m \times m$ matrix with its elements being either 1 or 0 . 
$B=\left[b_{1}, b_{2}, \ldots, b_{m}\right]^{t}$ with its element $b_{i}$ being the tolerance of the corresponding $i^{t h}$ dimensional chains,

$E=\left[e_{1}, e_{2}, \ldots, e_{m}\right]^{t}$ its element $e_{j}$ is called minimum economic tolerance for the $j^{\text {th }}$ working dimension.

The objective function (2) is to maximize the cumulative tolerance of each working dimension, which corresponds to assigning each tolerance to be as large as possible. Constraint set (3) reflects the dimensional chains of both stock removal and B/P dimensions, and has $\mathrm{m}$ inequalities because there are $\mathrm{m}$ dimensional chains. The sequence number of these stock removals may not be consecutive; however, this does not affect the linear programming formulation. Due to the principle of the tolerance stackup of a dimensional chain, the tolerances of the constituent links are always added together, whether a link is negative or positive. Constraint set (4) is required from minimum economic capability of each process sequence.

\section{DATA PROCESSING IMPLEMENTATION}

Next to the preceding study about the tolerance chart method, and with the purpose to simplify the task of the design office clerks, we have decided to automate this method by using the programming language $\mathrm{C}++$. The structure of the system we have conceived is presented in Fig. 2.

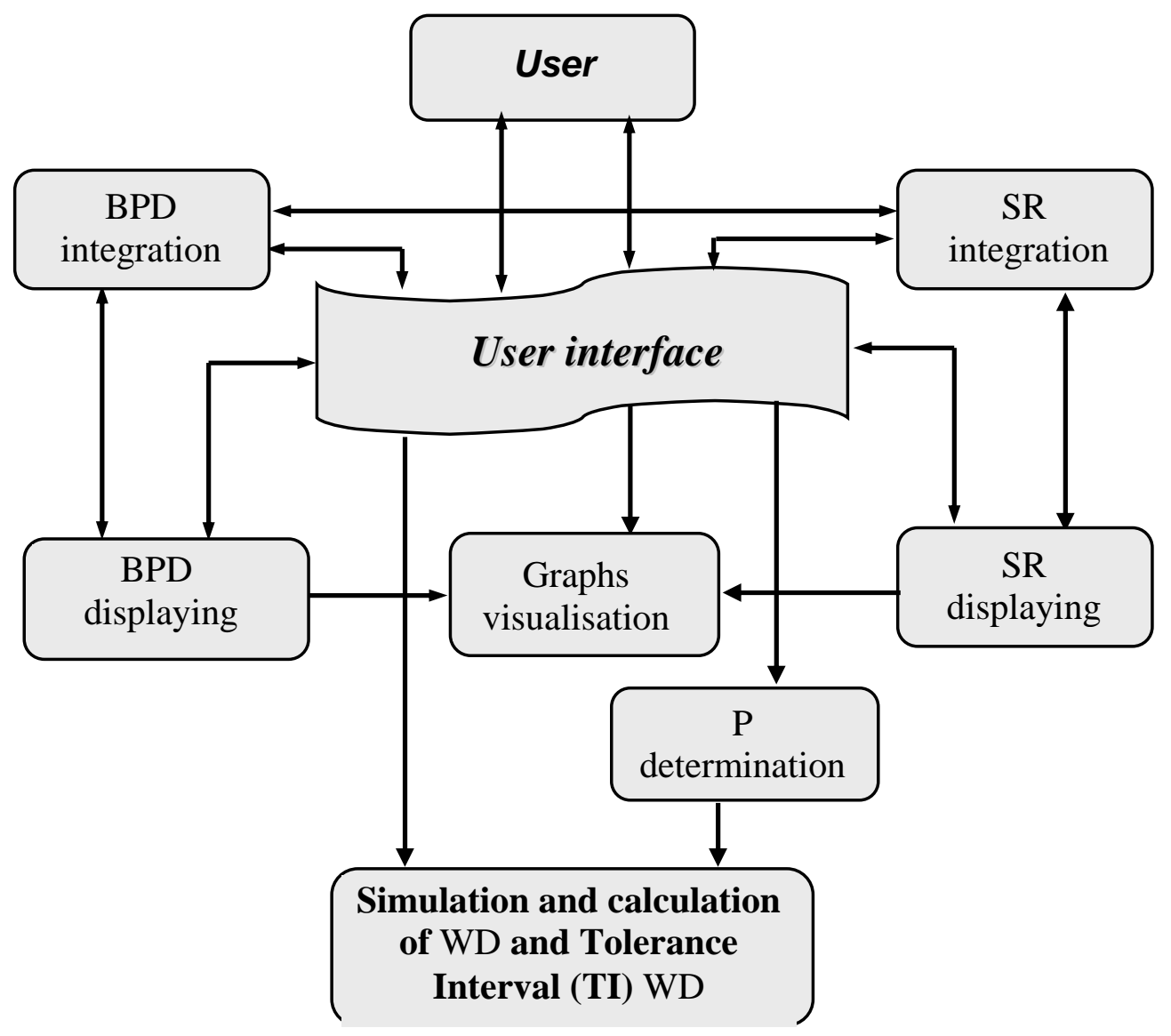

Figure 2: Architecture of the simulation data processing model.

In fast, this language architecture allows to easily integrate the data base provided by the design office and those proposed by the process office with the aid of the developed man- 
machine interface. The achieved program will allow presenting the three graphs already studied and which are: the BPD graph, the BPD and SR graph and the WD graph.

The simulation data processing tool allows visualizing - besides the 3 graphs, a file containing the BPD, another one containing the BPD and finally a last file which allows controlling the WD calculation and gives the values thus found from the provided conditions.

\section{APPLICATION EXAMPLE}

The part to study is a drive shaft whose definition drawing is given in Fig. 3. It has the function of bearing two turning grindstones driven by an engine.

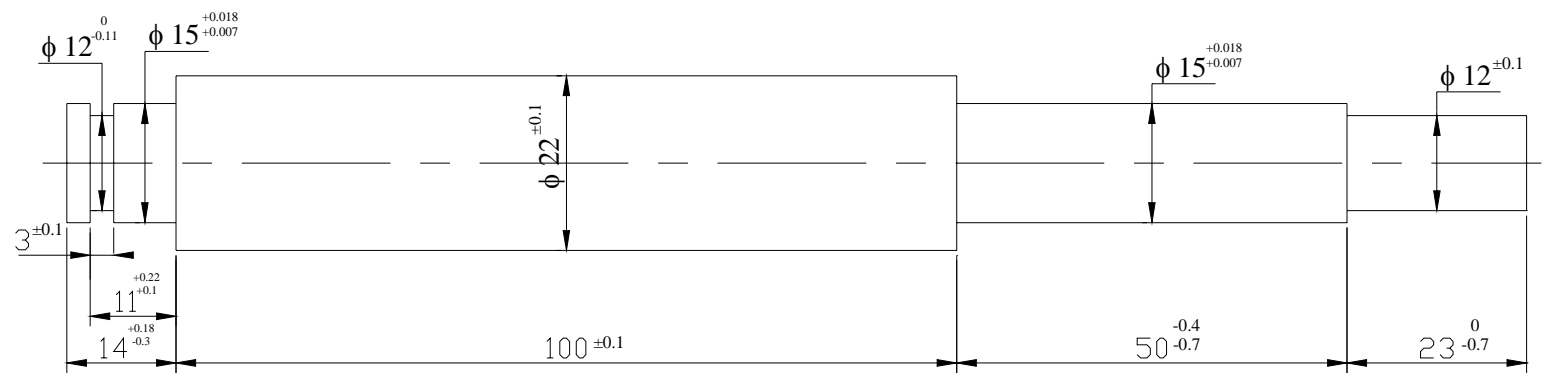

Figure 3: Drawing of the drive shaft.

\subsection{Process planning}

Table I shows the drive shaft process planning from a rough part which is a cylinder of 22 $\mathrm{mm}$ in diameter and $190 \mathrm{~mm}$ in length.

Table I: Process planning of the drive shaft.

\begin{tabular}{|c|c|}
\hline Designations & Sketch \\
\hline $\begin{array}{l}\text { Phase } 10 \text { : Turning } \\
\text { Positioning : long centering on the diameter } 22 \text { and a thrust on the surface } 7 . \\
\text { Machining: finishing machining of surfaces } 1 .\end{array}$ & 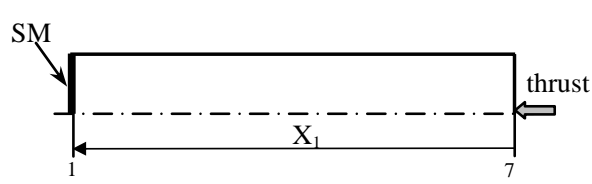 \\
\hline $\begin{array}{l}\text { Phase } 20 \text { : Turning } \\
\text { Positioning : long centering on the diameter } 22 \text { and a thrust on the surface } 1 . \\
\text { Machining: finishing machining of surfaces } 6 .\end{array}$ & $\underbrace{\mathrm{SM}}_{6}$ \\
\hline $\begin{array}{l}\text { Phase } 30 \text { : Turning } \\
\text { Positioning : long centering on the diameter } 22 \text { and a thrust on the surface } 6 . \\
\text { Machining: Rough machining of surfaces } 4 \text { ' and 5'. } \\
\text { Finishing machining of surfaces } 4 \text { and } 5 .\end{array}$ & ................ \\
\hline $\begin{array}{l}\text { Phase } 40 \text { : Turning } \\
\text { Positioning : long centering on the diameter } 15 \text { and a thrust on the surface } 4 . \\
\text { Machining: Rough machining of surfaces } 3 \text { '. } \\
\quad \text { Finishing machining of surfaces } 3 \text { and } 2 .\end{array}$ & 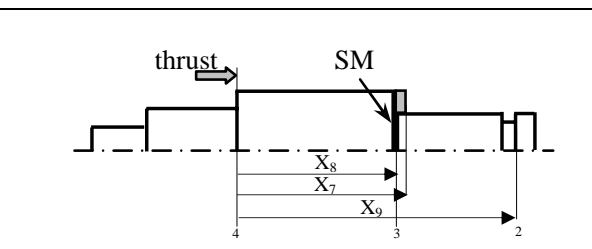 \\
\hline
\end{tabular}




\subsection{Results of simulation}

The drive shaft manufacturing is achieved by following the process planning already presented in Table I. In order to find the "working dimensions" between the machined surfaces, it is necessary to define the tolerance chart presented in Fig. 4.

In fact, we draw a table which illustrates the process planning, precising the number of the operations and numbering the dimensions accruing in the course of each operation.

These working dimensions are intended to be realized at the time of each operation, they are drawn successively as they occur during the machining.

These dimensions will be represented in the form of vectors having the support surface for origin and the machined surface for extremity. Each dimension represents one operation.

Then, we have to draw the first dimensional chain taking as reference dimension the first functional dimension, and follow on by drawing the other chains in the order of the functional dimensions and of the stock removals.

The identification of the different dimensional chains is quite easy to do basing on the generation of three graphs from the calculation sheet thus obtained and which is nothing but a theoretical representation of the tolerance chart.

A second table will be drawn (table BPD). The latter consists of two principal columns, one for the working dimensions, and the other is for the stock removals. The first column itself is made up of two columns, one of which is for the average values while the other is for the tolerance associated to that dimension.

The stock removals column includes, besides the average values, and the tolerance adjustment, a third column called "chain" containing the number of the different dimensions which intervene to form the chain of this stock removal, while respecting the sense of the dimension compared with the positive sense admitted.

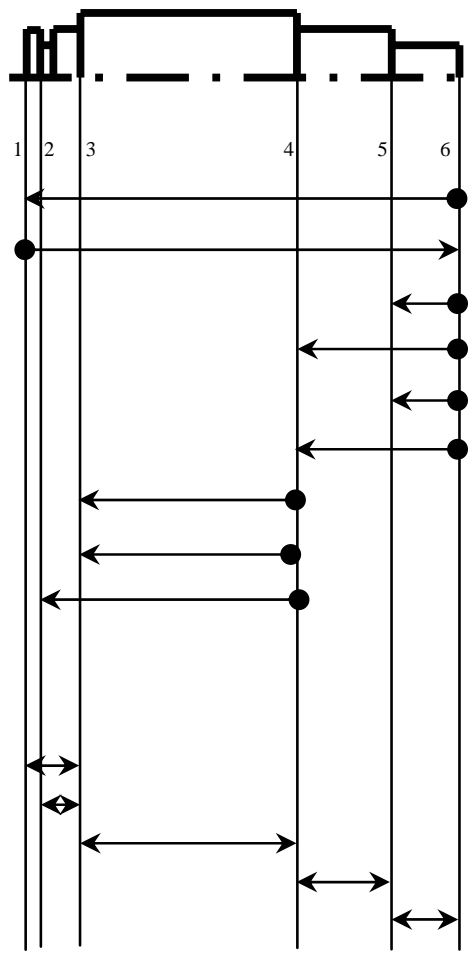

\begin{tabular}{|c|c|c|c|c|}
\hline \multicolumn{2}{|c|}{ Working Dim. } & \multicolumn{3}{|c|}{ Stock Removal } \\
\hline Mean & +/-Tol. & Mean & +/-Tol. & Chain \\
\hline $\mathrm{X} 1$ & & Rough & & \\
\hline $\mathrm{X} 2$ & & $1.4 \quad(\mathrm{Y} 6)$ & $+/-0.4$ & $1,-2$ \\
\hline X3 & & Rough & & \\
\hline $\mathrm{X} 4$ & & Rough & & \\
\hline X5 & & $2 \quad(\mathrm{Y} 7)$ & $+/-0.1$ & $3,-5$ \\
\hline X6 & & $2 \quad(Y 8)$ & $+/-0.1$ & $4,-6$ \\
\hline $\mathrm{X7}$ & & Rough & & \\
\hline $\mathrm{X} 8$ & & 2 (Y9) & $+/-0.1$ & $8,-7$ \\
\hline X9 & & Rough & & \\
\hline
\end{tabular}

\begin{tabular}{|c|c|c|}
\hline \multicolumn{3}{|c|}{ BPD } \\
\hline Nominal & Tolerance+/- & Chain \\
\hline $13.94 \mathrm{Y} 1$ & 0.24 & $2,-6,-8$ \\
\hline $11.16 \mathrm{Y} 2$ & 0.06 & $9,-8$ \\
\hline $100 \quad \mathrm{Y} 3$ & 0.1 & 8 \\
\hline $49.45 \mathrm{Y} 4$ & 0.15 & $6,-5$ \\
\hline 22.8 Y5 & 0.2 & 5 \\
\hline
\end{tabular}

Figure 4: Tolerance chart. 
In fact the dimensional chain must be identified for each working dimension and for each blueprint dimension as well as for any average value of stock removal.

It is to be noted that if the dimension is obtained directly from the rough piece, all you have to do is to mention it, in the box corresponding to that dimension in the column of stock removal. Otherwise you have to write down the stock removal value and the admitted tolerance.

Once all the boxes of the stock removal part are filled, all that remains is to mark the working dimensions and their tolerances. The latter are determined afterwards from the design office constraints and from the capability of the process used, and of the machine chosen.

In the following, we are going to present the results obtained by simulation on the software model.

From the design office conditions integrated through the interface of the BPD $\left(Y_{1}, Y_{2}, Y_{3}\right.$ $Y_{4}, Y_{5}$ ), we obtain the graph of the blueprint dimensions presented in Fig. 5 by simply pressing the control button « gr cbe ».

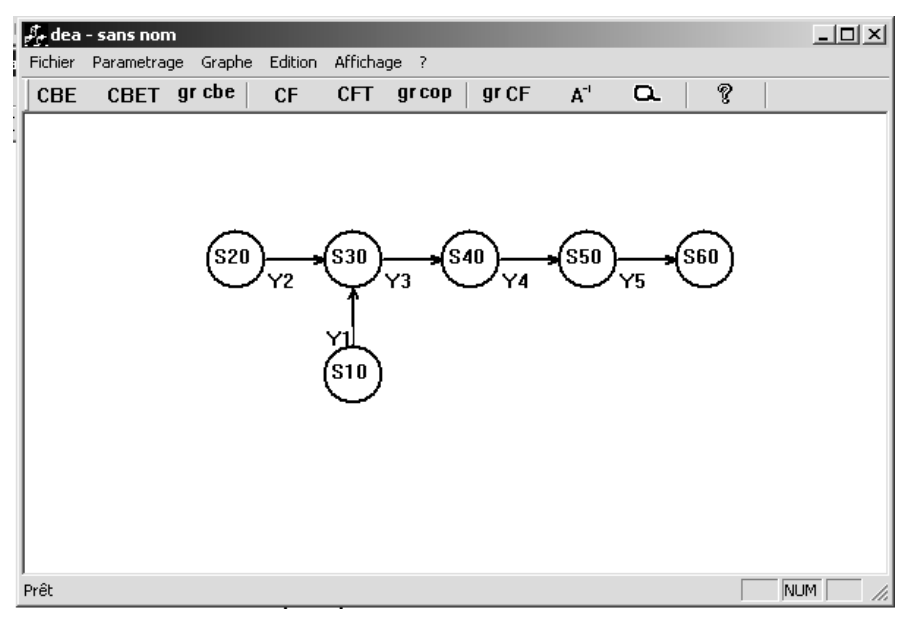

Figure 5: BPD Graph.

The representation of the design office conditions and those of the process office $\left(Y_{6}, Y_{7}\right.$, $\left.Y_{8}, Y_{9}\right)$ gives us the graph of Fig. 6 by pressing the control button « gr cop ».

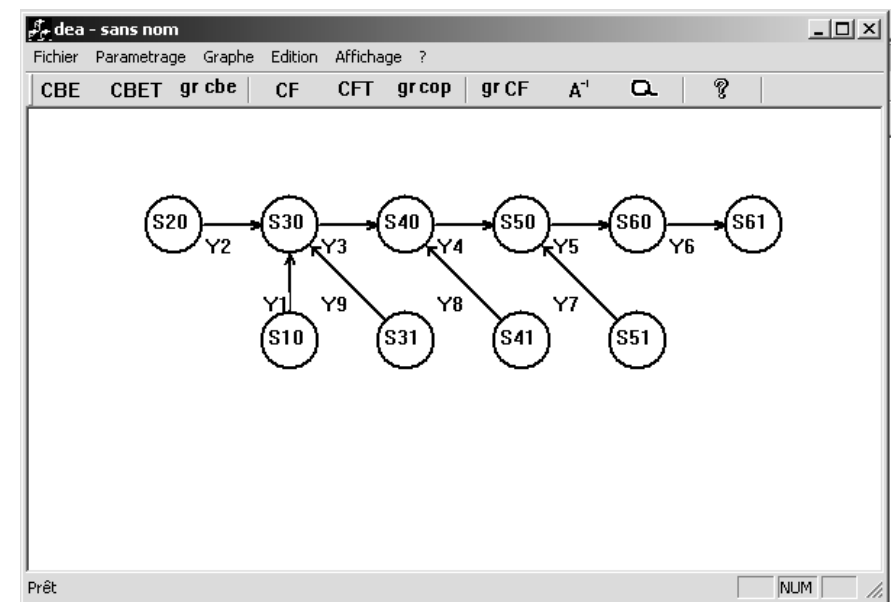

Figure 6: BPD Graph and SR.

The exploitation of the process office conditions by using the theories already presented, gives the graph WD of Fig. 7 by pressing on the control buttom « gr CF ». 


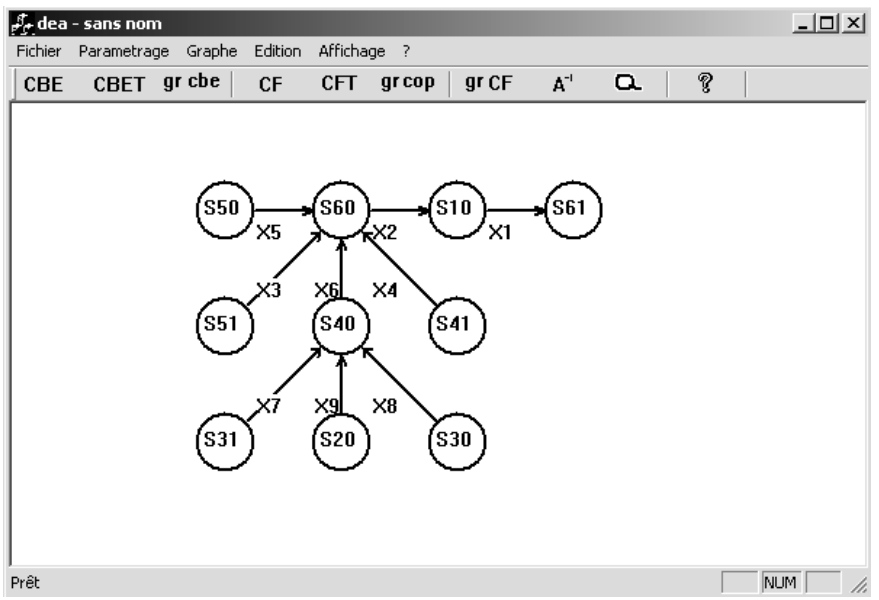

Figure 7: WD Graph.

Once the three graphs are generated, it is necessary to determine the dimensional chains in order to deduct the matrix $P$ linking the vector of the working dimensions $X_{i}$ to the vector of the functional dimensions $Y_{i}$. From the matrix $P^{-1}$ the data processing system can find the values of working dimensions given in Fig. 8.

Within the scope of this application, to find out the tolerances intervals of the working dimensions, we have supposed the values of the minimum economic tolerances and the weight of the tolerances following values:

$$
\begin{aligned}
& E=(0.05 ; 0.05 ; 0.05 ; 0.03 ; 0.03 ; 0.03 ; 0.03 ; 0.02 ; 0.015)^{t} \\
& B=(0.3 ; 0.25 ; 0.5 ; 0.15 ; 0.4 ; 0.15 ; 0.125 ; 0.025 ; 0.075)^{t} \\
& W=(1,1,4,1,4,2,2,2,3)
\end{aligned}
$$

The values of the tolerances intervals are calculated and given by the vector indicated in Fig. 8.

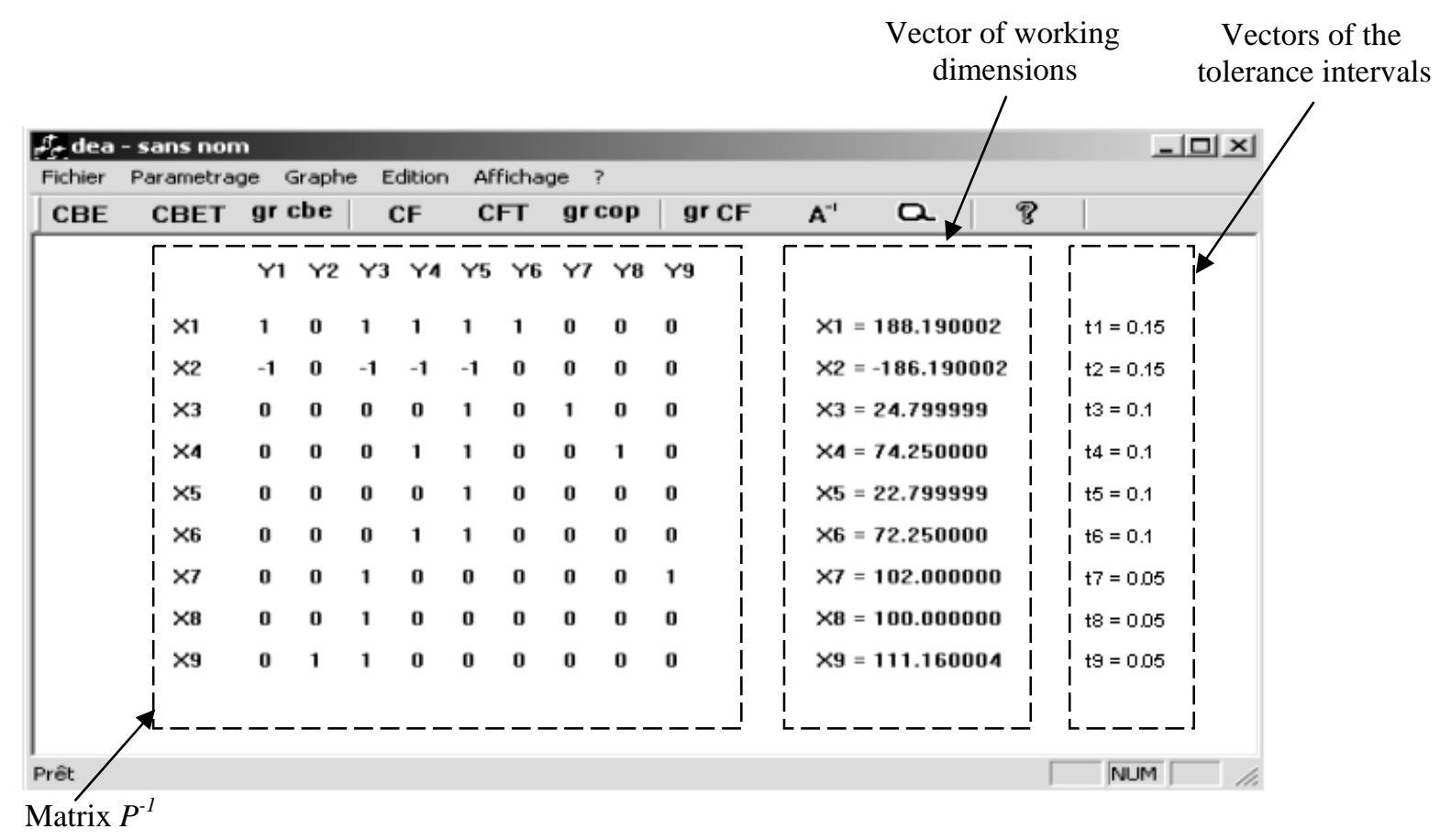

Figure 8: Calculation of the WD and tolerance intervals. 


\section{CONCLUSION}

This paper presents a computer-aided operational dimensioning system which is able to identify dimensional chains, assign tolerances and calculate mean sizes of working dimensions in a process plan without human interruption. The dimensional chains were found by the tree theoretical presentation, the tolerances were assigned by a linear programming model and the mean sizes of working dimensions were calculated by a linear equation set obtained from the dimensional chains. The calculation of all these working dimensions is based on the minimum economic tolerances and on the weight of tolerances.

The software tool which we have developed presents some industrial interests in the field of mechanical production. In fact, to calculate the working dimensions with their tolerances is a heavy task which is manually difficult. Hence, the necessity to have recourse to the computer aided manufacturing CAM.

\section{REFERENCES}

[1] Louati, J.; Ayadi, B.; Bouaziz, Z.; Haddar, M. (2005). Geometric Defaults Three-Dimensional Modelling to Optimize a Manufactured Part Setting, International Journal of Advanced Manufacturing Technology (in press)

[2] Anselmetti, B.; Bourdet, P. (1993). Optimization of a work piece considering production requirements, Computers in industry, Vol. 21, 23 - 34

[3] Linares, J. M. (1996). Contribution à l'étude de la cotation fonctionnelle par une approche systémique, Thèse, Institut National des Sciences Appliquées de Lyon, France

[4] Ping, J. I.; Mingyang, K. E.; Ahluwalia, R. S. (1995). Computer aided operational dimensioning for process planning, International Journal of Machine tools and Manufacture, Vol. 35, No. 10, $1353-1362$

[5] Salomons, O. W.; Poerink, H. J. J.; Haalboom, F. J.; van Slooten, F.; van Houten, F. J. A. M.; Kals, H. J. J. (1996). A computer aided tolerancing tool I: Tolerance specification, Computers in industry, Vol. 31, No. 2, 161-174

[6] Salomons, O. W.; Haalboom, F. J.; Poerink, H. J. J.; van Slooten, F.; van Houten, F. J. A. M.; Kals, H. J. J. (1996). A computer aided tolerancing tool II: Tolerance analysis, Computers in industry, Vol. 31, No. 2, 175-186

[7] Jami, J. S.; Yong, Y.; Bing-Chun, Z. (1998). Dimension and tolerance modelling and transformations in feature based design and manufacturing, Journal of Intelligent Manufacturing, Vol. 9, 475-488

[8] Yeo, S. H.; Ngoi, B. K. A.; Chen, H. (1998). Process sequence optimization based on a new costtolerance model, Journal of Intelligent Manufacturing, Vol. 9, 29-37

[9] Ping, J. I. (1999). An algebraic approach for dimensional chain identification in process planning, International Journal of Production and Research, Vol. 37, No. 1, 99-110

[10] Fainguelernt, D.; Weill, R.; Bourdet, P. (1986). Computer Aided Tolerancing and Dimensioning Process Planning, Annals of the CIRP, Vol. 35, No. 1, 381-386

[11] Villeneuve, F.; Vignat, F. (2003). 3D synthesis of manufacturing tolerances using a SDT approach, The $8^{\text {th }}$ CIRP International Seminar on Computer Aided Tolerancing, Charlotte, North Carolina, USA

[12] Whybrew, K.; Britton, G. A.; Robinson, D. F.; Sersuti-Anuwat, Y. (1990). A graph-theoretic approach to tolerance charting, International Journal of Advanced Manufacturing Technology, Vol. 5, 175-183 